\title{
Bounded and dissipative solutions of the Bouc-Wen model for hysteretic structural systems
}

\author{
Fayçal Ikhouane, Víctor Mañosa, José Rodellar
}

\begin{abstract}
The aim of this paper is to give the conditions on the hysteretic Bouc-Wen model so that (i) it has the property of being bounded input bounded output (BIBO), and (ii) it dissipates energy (or is passive). This study leads to a classification of the possible BIBO and dissipative (or passive) Bouc-Wen models.
\end{abstract}

\section{INTRODUCTION}

To describe the behavior of hysteretic processes several mathematical models have been proposed [11]: the Duhem model uses the property that a hysteretic system's output changes its character when the input changes direction; the Ishlinskii hysteresis operator has been proposed as a model for plasticity-elasticity and the Preisach model has been used for the modelling of electromagnetic hysteresis . A survey of the mathematical models for hysteresis may be found in [6]. Most works devoted to controlling systems with a continuous hysteresis have used the backlash model (see for example [9], [10]). As noted as early as in [5]: "Use of backlash to model [a magnetic] hysteresis element misrepresent the behavior about the origin, does not properly account for saturation, and introduces a dead zone that does not exist".

The objective of the present paper is to introduce an alternative simple model of a smooth hysteresis known as the Bouc-Wen model. Proposed in 1976, this model (a first-order nonlinear differential equation) has been used experimentally mainly in wood joints and structural systems (see [1] for example) and has remained largely unknown for the wide control community due mainly to the absence of an analytical study of this model. The aim of this paper is to fill this gap by giving the conditions on the Bouc-Wen model so that it holds the property of being bounded input bounded output (BIBO) and, moreover, it dissipates energy.

\footnotetext{
This work was supported by CICYT-Spain under project DPI200204018-C02-01. The first author acknowledges the support of the Spanish Ministry of Science and Technology through the "Ramón y Cajal" program. The second author acknowledges the partial support of the Government of Catalonia's grant 2001SGR-00173.

F. Ikhouane and J. Rodellar are with the Departament de Matemàtica Aplicada III, Universitat Politècnica de Catalunya, C/ Jordi Girona 1-3, 08034 - Barcelona, Spain faycal.ikhouane@upc.es, jose.rodellar@upc.es

V. Mañosa is with the Departament de Matemàtica Aplicada III, Universitat Politècnica de Catalunya, C/ Colom 1, 08222 - Terrassa, Spain victor.manosa@upc.es
}

\section{BIBO BOUC-WEN MODEL PROPERTIES}

\section{A. The model}

Consider a physical system with a hysteretic component that can be represented by a map $x(t) \mapsto \Phi_{s}(x(t), t)$, which is referred to as the "true" hysteresis. The so-called Bouc-Wen model [12] represents the true hysteresis in the following form:

$$
\begin{aligned}
\Phi_{B W}(x, t) & =\alpha k x(t)+(1-\alpha) D k z(t), \\
\dot{z} & =D^{-1}\left(A \dot{x}-\beta|\dot{x}||z|^{n-1} z-\gamma \dot{x}|z|^{n}\right),
\end{aligned}
$$

where $\dot{z}$ denotes the time derivative, and $n>1, D>0, k>0$ and $0<\alpha<1$ are parameters. The limit cases $n=1, \alpha=0$, $\alpha=1$ are treated in Appendix C.

This model was originally developed in the context of mechanical systems in which $x$ is a displacement and $\Phi$ is a restoring force. It represents the hysteretic force $\Phi_{s}(x, t)$ as the superposition of an elastic component $\alpha k x$ and a purely hysteretic component $(1-\alpha) k D z$, in which $D>0$ is the yield constant displacement and $\alpha \in(0,1)$ is the post to pre-yielding stiffness ratio. The hysteretic part involves a nondimensional auxiliary variable $z$ which is the solution of the nonlinear first order differential equation (2). In this equation, $A, \beta$ and $\gamma$ are nondimensional parameters which control the shape and the size of the hysteresis loop, while $n$ is a scalar that governs the smoothness of the transition from elastic to plastic response.

\section{B. Problem statement}

This study lies in the experimentally based premise that a true physical hysteretic element is BIBO, which means that, for any bounded input signal $x(t)$, the hysteretic response is also bounded. Thus the Bouc-Wen model $\Phi_{B W}$ should keep the $\mathrm{BIBO}$ property in order to be considered an adequate candidate to model real physical systems. Appendix A gives an example of a set of parameters $A, \beta, \gamma, n$ such that, for a particular bounded input $x(t)$, the corresponding output $\Phi_{B W}(x(t), t)$ given by the Bouc-Wen model (1)-(2) is unbounded. This means that, with this set of parameters, the Bouc-Wen model cannot describe the true hysteresis. This motivates the following problem:

Given the parameters $0<\alpha<1, k>0, D>0, A, \beta, \gamma$ and $n>1$, find the set of initial conditions $z(0)$ for which the Bouc-Wen model (1)-(2) is BIBO. Note that when this set is empty, this means that the Bouc-Wen model is not BIBO. The solution of this problem will lead to classify different 
sets of parameters and initial conditions and, additionally, to determine explicit bounds for the hysteretic variable $z(t)$.

\section{Classification of the BIBO Bouc-Wen models}

Let us introduce the following set:

$$
\Omega_{\alpha, k, D, A, \beta, \gamma, n}=\left\{z(0) \in \mathbb{R} \text { such that } \Phi_{B W}\right. \text { is BIBO }
$$

for all $C^{1}$ input signals $x(t)$ with fixed values of the parameters $\alpha, k, D, A, \beta, \gamma, n\}$.

The rest of the analysis is devoted to determining explicitly the set $\Omega_{\alpha, k, D, A, \beta, \gamma, n}$ as a function of the Bouc-Wen model parameters. Let $z(0)$ be an element of $\Omega_{\alpha, k, D, A, \beta, \gamma, n}$. Then, for any bounded $C^{1}$ input $x(t)$, the output $\Phi_{B W}(x(t), t)$ is bounded. This implies by equation (1) that the output $z(t)$ of the differential equation (2) should be bounded. This means that the set $^{1}$

$$
\begin{gathered}
\Omega_{A, \beta, \gamma, n}=\{z(0) \in \mathbb{R} \text { such that } z(t) \text { is bounded for } \\
\text { any } C^{1} \text { bounded input signal } x(t) \text { with fixed } \\
\text { values of the parameters } A, \beta, \gamma, n\}
\end{gathered}
$$

is such that $\Omega_{\alpha, k, D, A, \beta, \gamma, n} \subseteq \Omega_{A, \beta, \gamma, n}$. The inclusion in the other way is immediate, which shows that $\Omega_{\alpha, k, D, A, \beta, \gamma, n}=$ $\Omega_{A, \beta, \gamma, n}$. The importance of this equality stems for the fact that it is easier to determine the set $\Omega_{A, \beta, \gamma, n}$. Note that an empty set $\Omega_{A, \beta, \gamma, n}$ means that, with the chosen parameters $A$, $\beta, \gamma, n$, the Bouc-Wen model does not represent adequately the behavior of a real hysteretic system $\Phi_{s}$ (see Appendix A). We also define the following set:

$$
\begin{gathered}
\Omega_{A, \beta, \gamma, n}^{*}=\{z(0) \in \mathbb{R} \text { such that } z(t) \text { is bounded } \\
\text { for any } C^{1} \text { input signal } x(t) \text { with fixed } \\
\text { values of the parameters } A, \beta, \gamma, n\} .
\end{gathered}
$$

Note that $\Omega_{A, \beta, \gamma, n}^{\star} \subseteq \Omega_{A, \beta, \gamma, n}$. With the notations introduced above, the main results of this section are given below.

Theorem 1: Let $x(t), t \in[0, \infty)$ be a $C^{1}$ input signal and

$$
z_{0} \triangleq \sqrt[n]{\frac{A}{\beta+\gamma}} \text { and } z_{1} \triangleq \sqrt[n]{\frac{A}{\gamma-\beta}}
$$

Then, Table 1 holds.

$$
\text { Corollary 1: } \Omega_{A, \beta, \gamma, n}^{\star}=\Omega_{A, \beta, \gamma, n} \text {. }
$$

Corollary 1 means that the boundedness of the signal $z(t)$ depends only on the parameters $A, \gamma, \beta$ and $n$, while it is independent of the boundedness of the input signal $x(t)$. This fact is important for system control theory [2]: when $x(t)$ is a closed loop signal, we cannot assume a priori

\footnotetext{
${ }^{1}$ The correct notation would be $\Omega_{A, \beta, \gamma, n, D}$. However, we will see later that this set does not depend on the parameter $D$.
}

TABLE I

ClassificATION OF THE BIBO BOUC-WEN MODELS

\begin{tabular}{|l|l|l|l|l|}
\hline \multicolumn{2}{|c|}{ Case } & $\Omega_{A, \beta, \gamma, n}$ & $|z(t)|$ bound & Class \\
\hline \multirow{3}{*}{$A>0$} & $\beta+\gamma>0, \beta-\gamma \geq 0$ & $\mathbb{R}$ & $\max \left(|z(0)|, z_{0}\right)$ & I \\
\cline { 2 - 5 } & $\beta-\gamma<0, \beta \geq 0$ & {$\left[-z_{1}, z_{1}\right]$} & $\max \left(|z(0)|, z_{0}\right)$ & II \\
\hline \multirow{2}{*}{$A<0$} & $\beta-\gamma>0, \beta+\gamma \geq 0$ & $\mathbb{R}$ & $\max \left(|z(0)|, z_{1}\right)$ & III \\
\cline { 2 - 5 } & $\beta+\gamma<0, \beta \geq 0$ & {$\left[-z_{0}, z_{0}\right]$} & $\max \left(|z(0)|, z_{1}\right)$ & IV \\
\hline$A=0$ & $\beta+\gamma \geq 0, \beta-\gamma \geq 0$ & $\mathbb{R}$ & $|z(0)|$ & V \\
\hline \multicolumn{2}{|c|}{ all other cases } & $\emptyset$ & & \\
\hline
\end{tabular}

that it is bounded. The fact that $\Omega_{A, \beta, \gamma, n}^{\star}=\Omega_{A, \beta, \gamma, n}$ shows that for every input signal $x(t)$ (under the only assumption that it is $C^{1}$ ), the output $z(t)$ is always bounded if the set $\Omega_{A, \beta, \gamma, n}$ is non-empty, and if $z(0) \in \Omega_{A, \beta, \gamma, n}$.

Corollary 2: In all cases where a non-empty set $\Omega_{A, \beta, \gamma, n}$ exists, the parameter $\beta$ is non-negative.

Starting from physical considerations, a result close to that of Corollary 2 has been drawn in [8] using the fact that thermodynamic laws require $\beta>0$ for the energy to be dissipated through each hysteretic cycle.

Proof: First we check that the differential equation (2) has a unique solution. Equation (2) may be seen as a nonautonomous locally Lipschitz system (as $n>1$ ) where the dependence on time is continuous. Thus, by [4, Theorem 2.2] a unique solution of (2) does exist. We consider the following three cases: $A>0, A<0$ and $A=0$.

We focus first on the case $A>0$. Consider the following three possibilities:

$$
\begin{aligned}
& P_{1}: \beta+\gamma>0 \text { and } \beta-\gamma \geq 0, \\
& P_{2}: \beta+\gamma>0 \text { and } \beta-\gamma<0, \\
& P_{3}: \beta+\gamma \leq 0 .
\end{aligned}
$$

Let us focus on the case $P_{1}$. We consider the Lyapunov function candidate $V(t)=z(t)^{2} / 2$. Its derivative takes different forms depending on the signs of $\dot{x}$ and $z$. Indeed, setting $Q_{1}=\{\dot{x} \geq 0$ and $z \geq 0\}$, and denoting $\dot{V}_{\mid Q_{1}}$ as the expression of the derivative of the function $V$ over the set $Q_{1}$, we have $\dot{V}_{\mid Q_{1}}=z \dot{x} D^{-1}\left(A-(\beta+\gamma) z^{n}\right)$. Thus $\dot{V}_{\mid Q_{1}} \leq 0$ for $z \geq z_{0}$. Also, if we set $Q_{2}=\{\dot{x} \geq 0$ and $z \leq 0\}$, we have $\dot{V}_{\mid Q_{2}}=z \dot{x} D^{-1}\left(A+(\beta-\gamma)|z|^{n}\right)$. In this case, $\dot{V}_{\mid Q_{2}} \leq 0$ for all values of $z$. The same conclusion is drawn in the case of $Q_{3}=\{\dot{x} \leq 0$ and $z \geq 0\}$, since $\dot{V}_{\mid Q_{3}}=z \dot{x} D^{-1}\left(A+(\beta-\gamma) z^{n}\right)$. Finally, taking $Q_{4}=\{\dot{x} \leq 0$ and $z \leq 0\}$, we get $\dot{V}_{\mid Q_{4}}=$ $z \dot{x} D^{-1}\left(A-(\beta+\gamma)|z|^{n}\right)$. Thus, $\dot{V}_{\mid Q_{4}} \leq 0$ for $|z| \geq z_{0}$. We then conclude that, for all the possibilities of the signs of $\dot{x}$ and $z$, we have $\dot{V} \leq 0$ for all $|z| \geq z_{0}$. By [4, Theorem 4.10] 2 we conclude that $z(t)$ is bounded for every continuous function $\dot{x}(t)$ and every initial condition $z(0)$ which means that $\Omega_{A, \beta, \gamma, n}^{\star}=\mathbb{R}$. Since $\Omega_{A, \beta, \gamma, n}^{\star} \subseteq \Omega_{A, \beta, \gamma, n}$, this implies that $\Omega_{A, \beta, \gamma, n}^{\star}=\Omega_{A, \beta, \gamma, n}=\mathbb{R}$. The bounds on $z(t)$ can be derived from [4, Theorem 4.10] as follows:

\footnotetext{
${ }^{2}$ The function $\alpha_{3}$ which appears in theorem 4.10 of [4] is zero in our case. It is not class $\mathscr{K}$. This means that the time $t_{1}$ of equation 4.22 of [4] may be infinite and that all what we can say about $x(t)$ in [4] is that $\|x(t)\| \leq \max \left(\left\|x\left(t_{0}\right)\right\|, \alpha_{1}^{-1}\left(\alpha_{2}(\mu)\right)\right.$ which is exactly what we use.
} 
i) If the initial condition of $z$ is such that $|z(0)| \leq z_{0}$ then $|z(t)| \leq z_{0}$ for all $t \geq 0$

ii) If the initial condition of $z$ is such that $|z(0)| \geq z_{0}$ then $|z(t)| \leq|z(0)|$ for all $t \geq 0$.

We now turn to the case $P_{2}$. Again, the derivative of $V(t)$ depends on the signs of $\dot{x}$ and $z$. Indeed, $\dot{V} \leq 0$ in the following regions:

$$
\begin{aligned}
& \left\{\dot{x} \geq 0 \text { and } z \geq 0 \text { and } z \geq z_{0}\right\}, \\
& \left\{\dot{x} \geq 0 \text { and } z \leq 0 \text { and }|z| \leq z_{1}\right\}, \\
& \left\{\dot{x} \leq 0 \text { and } z \geq 0 \text { and } z \leq z_{1}\right\}, \\
& \left\{\dot{x} \leq 0 \text { and } z \leq 0 \text { and }|z| \geq z_{0}\right\} .
\end{aligned}
$$

Then, from (7)-(10) we conclude that: if $z_{1} \geq z_{0}$ (that is, when $\beta \geq 0$ ), then $\dot{V} \leq 0$ for every $z_{0} \leq|z| \leq z_{1}$ independently of the sign of $\dot{x}$. By [4, theorem 4.10] we conclude that $z(t)$ is bounded for every continuous function $\dot{x}(t)$ and any initial state $z(0)$ such that $|z(0)| \leq z_{1}$. This means that $\left[-z_{1}, z_{1}\right] \subseteq \Omega_{A, \beta, \gamma, n}^{\star}$. Now, take $z(0) \notin\left[-z_{1}, z_{1}\right]$; we claim that there exists a bounded $C^{1}$ signal $x(t)$ such that the corresponding signal $z(t)$ is unbounded. The construction of such a signal is done in Appendix VI, which means that $z(0) \notin \Omega_{A, \beta, \gamma, n}$. This implies that $\Omega_{A, \beta, \gamma, n} \subseteq\left[-z_{1}, z_{1}\right]$. Since $\Omega_{A, \beta, \gamma, n}^{\star} \subseteq \Omega_{A, \beta, \gamma, n}$, we get $\Omega_{A, \beta, \gamma, n}^{\star}=\Omega_{A, \beta, \gamma, n}=\left[-z_{1}, z_{1}\right]$. Using [4, Theorem 4.10] we can obtain the following bound: $|z(t)| \leq \max \left(|z(0)|, z_{0}\right)$. If $\beta<0$, then for each initial condition $z(0)$ it is possible to construct a bounded $C^{1}$ signal $x(t)$ as in Appendix VI, such that the corresponding signal $z(t)$ is unbounded. This means that $\Omega_{A, \beta, \gamma, n}=\emptyset$. Since $\Omega_{A, \beta, \gamma, n}^{\star} \subseteq \Omega_{A, \beta, \gamma, n}$, this implies that $\Omega_{A, \beta, \gamma, n}^{\star}=\Omega_{A, \beta, \gamma, n}=\emptyset$.

We now move to the case $P_{3}$. An analysis similar to that of Appendix VI shows that $\Omega_{A, \beta, \gamma, n}=\emptyset$, which implies that $\Omega_{A, \beta, \gamma, n}^{\star}=\Omega_{A, \beta, \gamma, n}=\emptyset$.

The cases $A<0$ and $A=0$ can be treated in a similar way, which ends the proof of Theorem 1 .

Note that the case $\beta+\gamma>0, \beta-\gamma<0$ and $\beta \geq 0$ simplifies to $\beta-\gamma<0$ and $\beta \geq 0$ since the former inequality is implied by the latter two. Corollary 1 follows immediately from the proof of Theorem 1, while Corollary 2 follows from Table 1.

\section{UNCERTAINTY ISSUES}

In Section II-C we have seen that the Bouc-Wen model does not represent adequately a physical hysteretic system if the set $\Omega_{A, \beta, \gamma, n}$ is empty. When the set $\Omega_{A, \beta, \gamma, n}$ is not empty, we have determined it as an explicit function of the model parameters and we have also determined explicitly an upper bound on the solution $z(t)$. In practice, the model parameters are not known exactly: tuning or identification procedures allow reasonably the determination of approximate values of these parameters [7]. Let us consider a generic parameter $p \in\{A, \beta, \gamma, n\}$ and consider that the identification leads to the knowledge of minimum and maximum values such that $p_{\min } \leq p \leq p_{\max }$. Since $n>1$ and $\beta \geq 0$ (see Corollary 2 of Section II-C), we should have $n_{\min } \geq 1$ and $\beta_{\text {min }} \geq$ 
0 . Our task in this section is to determine a computable interval contained in the set $\Omega_{A, \beta, \gamma, n}$ and a computable upper bound on the solution $z(t)$ of the differential equation (2) for different cases in which the Bouc-Wen model parameters are defined as intervals. For reasons that will be clear in Section $\mathrm{V}$, we consider only the case where $A>0$, that is we consider in this part the Bouc-Wen models that belong to the classes I and II of Table 1.

We define the following constants:

$$
\begin{aligned}
& \underline{z}_{1}=\min \left(\sqrt[n \max ]{\left|\frac{A_{\min }}{\gamma_{\text {max }}-\beta_{\min }}\right|}, \sqrt[n]{\min } \sqrt{\left|\frac{A_{\min }}{\gamma_{\max }-\beta_{\min }}\right|}\right), \\
& \bar{z}_{0}=\max \left(\sqrt[n_{\max }]{\left|\frac{A_{\max }}{\gamma_{\min }+\beta_{\min }}\right|}, \sqrt[n]{\min } \sqrt{\left|\frac{A_{\max }}{\gamma_{\min }+\beta_{\min }}\right|}\right) .
\end{aligned}
$$

The analysis involve lengthy but straightforward calculations. The results are summarized in Table II. Some interpretations are made below. For example, if $A_{\min }>0$, $\beta_{\min }+\gamma_{\min }>0$ and $\beta_{\min }-\gamma_{\max }<0$, then a computable subset of $\Omega_{A, \beta, \gamma, n}$ is $\left[-\underline{z}_{1}, \underline{z}_{1}\right]$. The solution $z(t)$ is bounded and a computable upper bound on $z(t)$ is $\max \left(|z(0)|, \bar{z}_{0}\right)$. Consider now the case $A_{\min }>0$ and $\beta_{\max }+\gamma_{\max } \leq 0$. The set $\Omega_{A, \beta, \gamma, n}$ is empty, which means that the parameters do not correspond to a BIBO Bouc-Wen model. When $A_{\min }>0$ and $\beta_{\min }+\gamma_{\min } \leq 0<\beta_{\max }+\gamma_{\max }$, we cannot know from the available information if the set $\Omega_{A, \beta, \gamma, n}$ is empty or not. Thus, it is not possible to determine neither a computable subset of $\Omega_{A, \beta, \gamma, n}$ nor a computable upper bound on $|z(t)|$. This means that we should perform a more accurate identification of the parameters.

Note that the process of refining the identification procedure is always finite whenever the set $\Omega_{A, \beta, \gamma, n}$ is non-empty. Indeed, take series of values $\left\{p_{\min , k}, p_{\max , k}\right\}$ such that $p_{\min , k} \leq p_{\min , k+1} \leq p \leq p_{\max , k+1} \leq p_{\max , k}$ and $\lim _{k \rightarrow \infty} p_{\min , k}=\lim _{k \rightarrow \infty} p_{\max , k}=p$, where $p$ is any of the parameters $A, \beta, \gamma$ or $n$. Then there exists some integer $N$ such that $A \geq A_{\min , N}>0$. If the process of refining the identification were infinite, we would have from Table II: $\beta_{\min , k}+\gamma_{\min , k} \leq 0<\beta_{\max , k}+\gamma_{\max , k}$ for all $k \geq N$. Thus, taking the limit for $k \rightarrow \infty$, we get $\beta+\gamma=0$. It can be checked from Table 1 that this case corresponds to an empty set $\Omega_{A, \beta, \gamma, n}$. This means that, once the Bouc-Wen model is $\mathrm{BIBO}$, the process of refining the identification procedure leads in a finite number of steps to a computable subset of the set $\Omega_{A, \beta, \gamma, n}$ and a computable upper bound on $|z(t)|$.

\section{FREE MOTION OF AN HYSTERETIC STRUCTURAL SYSTEM}

\section{A. Problem statement}

We consider a structural isolation scheme, as illustrated in Figure 1, which is modelled as 1 degree-of-freedom system with mass $m>0$ and viscous damping $c>0$ plus a restoring force $\Phi$ characterizing a hysteretic behavior of the isolator material.



(a)

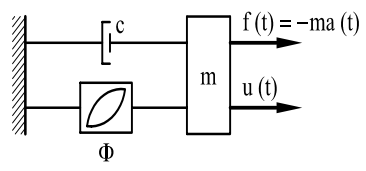

(b)
Fig. 1. Hysteretic isolation scheme (a) and its physical model (b).

This system is described by the second order differential equation

$$
m \ddot{x}+c \dot{x}+\Phi(x, t)=f(t)
$$

with initial conditions $x(0)$ and $\dot{x}(0)$ and excited by a force $f(t)$, like the one of the form $-m a(t)$ in the case of an earthquake with ground acceleration $a(t)$. The restoring force is assumed to be described by the Bouc-Wen model:

$$
\begin{aligned}
\Phi(x, t) & =\alpha k x(t)+(1-\alpha) D k z(t), \\
\dot{z} & =D^{-1}\left[A \dot{x}-\beta|\dot{x}||z|^{n-1} z-\gamma \dot{x}|z|^{n}\right],
\end{aligned}
$$

where $n>1, D>0, k>0$ and $0<\alpha<1$. The purpose of this section is the study of the free motion of system (11)-(13), that is with $f(t)=0$, to analyze its asymptotic trajectories. This analytical characterization will be used to reveal the energy dissipation properties of system (11)-(13) in Section V.

\section{B. Asymptotic trajectories}

The main result in this section is given in the following theorem.

Theorem 2: For every initial conditions $x(0) \in \mathbb{R}, \dot{x}(0) \in$ $\mathbb{R}$ and $z(0) \in \Omega_{A, \beta, \gamma, n} \neq \emptyset$, the following holds:

(a) For all the classes I-V of Table 1 , the signals $x(t), \dot{x}(t)$ and $z(t)$ are bounded and $C^{1}$.

(b) Assume that the Bouc-Wen model belongs to the classes I or II. Then, there exist constants $x_{\infty}$ and $z_{\infty}$, which depend only on the Bouc-Wen model parameters $(\alpha, D, k, A, \beta, \gamma, n)$, the system parameters $(m, c)$ and the initial conditions $(x(0), \dot{x}(0), z(0))$, such that

$$
\begin{aligned}
\lim _{t \rightarrow \infty} x(t) & =x_{\infty} \\
\lim _{t \rightarrow \infty} z(t) & =z_{\infty} \\
\alpha x_{\infty}+(1-\alpha) D z_{\infty} & =0 .
\end{aligned}
$$

Furthermore, we have

$$
\dot{x} \in L_{1}([0, \infty)) \quad \text { and } \quad \lim _{t \rightarrow \infty} \dot{x}(t)=0 .
$$

Proof: see [3].

\section{ENERGY DISSIPATION ANALYSIS}

The objective of this section is the analytical study of the energy dissipation mechanism in the system (11)-(13). As in Section IV-B, it is assumed that the Bouc-Wen model 
parameters are such that a non-empty set $\Omega_{A, \beta, \gamma, n}$ exists and that $z(0) \in \Omega_{A, \beta, \gamma, n}$. Write the system (11)-(13) as

$$
m \ddot{x}+c \dot{x}+\alpha k x+(1-\alpha) D k z=0
$$

where $z$ is the solution of the differential equation (2). At each instant $t$, the total energy $E(t)$ of (18) is the sum of its kinetic energy $\frac{1}{2} m \dot{x}(t)^{2}$ and its potential elastic energy $\frac{1}{2} \alpha k x(t)^{2}$. That is

$$
E(t)=\frac{1}{2} m \dot{x}(t)^{2}+\frac{1}{2} \alpha k x(t)^{2} .
$$

Consider the system (11)-(13) with $x(0)=0, \dot{x}(0)=0$ and assume that $z(0) \neq 0$. By continuity of the solutions of (11)-(13), the signal $z(t)$ will be nonzero at least during some time interval $\left[0, t_{1}\right)$. This implies by equation (18) that in the time interval $\left(0, t_{1}\right)$ the signals $x(t)$ and $\dot{x}(t)$ are not identically zero. In this case, the Bouc-Wen model has delivered non-identically zero signals $x$ and $\dot{x}$ starting from zero initial conditions $x(0)=0$ and $\dot{x}(0)=0$. This will unlikely be the case for the real hysteresis as, in general, the coordinates are chosen in such a way that the point with $x(0)=0$ and $\dot{x}(0)=0$ is an equilibrium position for the real hysteretic system under free motion. This means that the Bouc-Wen model cannot describe adequately the true hysteresis if $z(0)$ is not set to zero.

With $z(0)$ set to zero, a Bouc-Wen model is uniquely defined by its parameters $A, \beta, \gamma, n, D, \alpha, k$. Thus, each class of Table 1 can be seen as a set of allowable parameters, for example :

$$
\begin{aligned}
\text { Class I }= & \left\{(A, \beta, \gamma, n, D, \alpha, k) \in \mathbb{R}^{7}\right. \text { such that } \\
& A>0, \beta+\gamma>0, \beta-\gamma \geq 0, n>1, \\
& D>0,0<\alpha<1, k>0\} .
\end{aligned}
$$

We introduce some definitions.

Definition 1: The Bouc-Wen model defined by its parameters $(A, \beta, \gamma, n, D, \alpha, k)$ is said to be asymptotically dissipative if for every $m>0, c>0$ and every initial conditions $(x(0), \dot{x}(0))$ we have $E(\infty)<E(0)$ whenever $E(0) \neq 0$.

Definition 2: A class of the Table 1 is said to be asymptotically dissipative if all its elements are asymptotically dissipative.

Definition 3: The Bouc-Wen model defined by its parameters $(A, \beta, \gamma, n, D, \alpha, k)$ is said to generate energy if there exist some $m>0, c>0$, some initial condition $(x(0), \dot{x}(0))$ and some finite time $t_{0}$ such that $E\left(t_{0}\right)>E(0)$.

A Bouc-Wen model that generates energy is highly undesirable as it cannot represent the real passive hysteretic systems.

With the definitions above we now state the main result of this section.
Theorem 3: Consider the classes I-V of Table 1. Then, we have the following:

(i) The classes I and II are asymptotically dissipative.

(ii) The classes III and IV contain an infinite number of elements that generate energy.

(iii) For the class $\mathrm{V}$, the hysteretic part of the model is always zero.

Proof: see [3].

The results of Theorem 3 are illustrated in Figure 2.



Fig. 2. Classification of the possible BIBO and dissipative Bouc-Wen models.

\section{CONCLUSION}

This paper has presented a classification of the possible Bouc-Wen models in terms of their BIBO and energy dissipation properties. It has been shown that only five classes I-V of Bouc-Wen models are BIBO. The asymptotic behavior of a second order mechanical/structural hysteretic system represented by the Bouc-Wen model has been analyzed. It has been shown that, for all the five classes, the displacement of the mass and its velocity are bounded. Furthermore, for the classes I and II, the displacement of the mass goes asymptotically to a constant, the restoring force goes to zero, the velocity of the mass is in $L_{1}$ and goes to zero asymptotically.

This study led to the analysis of the energy dissipation properties of the five BIBO classes of Bouc-Wen models. It has been shown that the classes I and II are indeed asymptotically dissipative and thus may represent the physical behavior of a true hysteresis. The classes III and IV have been shown to contain an infinite number of elements which generates energy. This means that both classes are of little practical interest. The remaining class $\mathrm{V}$ has been shown to be irrelevant in practice since the hysteretic part of the model remains equal to zero and thus cannot describe an hysteretic behavior.

\section{APPENDIX A}

In this section, we present an example of a Bouc-Wen model which is not BIBO. Consider the Bouc-Wen model 
given by the following parameters: $D=1, A=1, \beta=0.5$, $\gamma=-1.5$ and $n=2$. Take $z(0)=0$ and define the bounded input signal $x(t)=\frac{\pi}{2} \sin (t)$. The corresponding derivative is $\dot{x}(t)=\frac{\pi}{2} \cos (t)$, which is also bounded. For $0 \leq t \leq \frac{\pi}{2}$ we have $\dot{x}(t) \geq 0$. This implies that the Bouc-Wen model (2) can take only one of the two forms:

$$
\begin{aligned}
& \dot{z}=\dot{x}\left(1+z^{2}\right) \quad \text { for } z \geq 0, \\
& \dot{z}=\dot{x}\left(1+2 z^{2}\right) \quad \text { for } z \leq 0 .
\end{aligned}
$$

In both cases (21) and (22) it is $\dot{z} \geq 0$ for $0 \leq t \leq \frac{\pi}{2}$, which implies that $z(t)$ is a non-decreasing function. Since $z(0)=0$, this means that $z(t) \geq 0$, so that $\dot{z}$ is given by (21). Integrating (21) we obtain

$$
\int \frac{d z}{1+z^{2}}=\int d x
$$

which gives $\arctan (z)=x$, since $z(0)=0$ and $x(0)=0$. This implies that $z(t)=\tan (x(t))$. Observe that $\lim _{t \rightarrow \pi / 2} z(t)=+\infty$. Thus, by equation (1), the $C^{1}$ bounded input signal $x(t)$ will give rise to an unbounded output $\Phi_{B W}(x, t)$. A similar construction can be done for any initial condition $z(0) \neq 0$.

\section{APPENDIX B}

Assume that $\beta-\gamma<0, \beta \geq 0$. The goal of this section is to construct a $C^{1}$ bounded input signal $x(t)$ such that the corresponding signal $z(t)$ is unbounded for any initial condition $z(0)$ such that $|z(0)|>z_{1}$. Take $z(0)>z_{1}$ (the construction is similar in the case $\left.z(0)<-z_{1}\right)$. Define the signal

$$
\dot{x}=\frac{D}{A+(\beta-\gamma) z^{n}} .
$$

Since $z(0)>z_{1}>0$, we have $A+(\beta-\gamma) z(0)^{n}<0$, which means that the solution $z(t)$ of the differential equation (2) is well defined at least during some time interval $\left[0, t_{1}\right)$ in which we have $z(t)>z_{1}$. For $0 \leq t<t_{1}$ we have $z>0$ and $\dot{x}<0$. Thus equation (2) reduces to

$$
\dot{z}=D^{-1} \dot{x}\left(A+(\beta-\gamma) z^{n}\right) \text {. }
$$

Combining equations (24) and (25), it follows that

$$
\dot{z}=1
$$

Integrating (26) gives for $0 \leq t<t_{1}$ :

$$
\begin{aligned}
z(t) & =t+z(0), \\
\dot{x}(t) & =\frac{D}{A+(\beta-\gamma)(t+z(0))^{n}} .
\end{aligned}
$$

The function $z(t)$ is increasing, so that the conditions of existence of $\dot{x}$ in (24) and (28) are satisfied for any $t \geq 0$. This means that $t_{1}=\infty$, that is $\dot{x}$ is well defined for all $t \geq 0$ and a solution of the differential equation (2) (given by (27)) exists over $t \in \mathbb{R}_{+}$. From equation (28), it follows that $\dot{x} \in L_{1}$ as $n>1$. This implies that $x(t)$ goes to a finite limit as $t$ goes to infinity, which means that $x(t)$ is bounded. Thus we have constructed a bounded $C^{1}$ signal $x(t)$ with an unbounded corresponding signal $z(t)$.

\section{APPENDIX C}

In this appendix we analyze the following limit cases for the Bouc-Wen model parameters: $n=1, \alpha=0, \alpha=1$.

\section{C1. The limit case $n=1$}

The differential equation (2) remains locally Lipschitz for $n=1$, however the signal $\dot{x}$ constructed in Appendix $\mathrm{B}$ is no longer in $L_{1}$ so that the result of that appendix does not hold. This means that in Table 1 the expressions given for $\Omega_{A, \beta, \gamma, n}$ are only subsets of the whole set $\Omega_{A, \beta, \gamma, n}$. With this simple observation, Table 1 holds also for the case $n=1$. For example, for the class II, a subset of $\Omega_{A, \beta, \gamma, n}$ is given by $\left[-z_{1}, z_{1}\right]$; and for $z(0) \in\left[-z_{1}, z_{1}\right]$, an upper bound on $|z(t)|$ is given by $\max \left(|z(0)|, z_{0}\right)$ as indicated in Table 1 . Theorem 2 holds for $n=1$ with the only modification that $z(0)$ should belong to the subset of $\Omega_{A, \beta, \gamma, n}$ given by Table 1 (and not to the whole set $\Omega_{A, \beta, \gamma, n}$ ). Theorem 3 holds as $z(0)=0$.

\section{C2. The limit case $\alpha=1$}

For $\alpha=1$ the hysteretic part in equation (1) is zero so that the system (18) is linear and thus does not represent a hysteretic nonlinearity.

\section{C3. The limit case $\alpha=0$}

Table 1 holds for the case $\alpha=0$. However, Theorems 2 and 3 do not hold necessarily (see [3] for a proof).

\section{REFERENCES}

[1] G. C. Foliente. Hysteresis modelling of wood joints and structural systems. ASCE Journal of Structural Engineering, 121(6), pp. 10131022, 1995.

[2] F. Ikhouane, V. Mañosa and J. Rodellar. Adaptive control of an hysteretic structural system. Submitted to Automatica, 2003.

[3] F. Ikhouane, V. Mañosa and J. Rodellar. Input-output and energy dissipation properties of the hysteretic Bouc-Wen Model. Submitted to SIAM. J. of Control and Optimization, 2003.

[4] H. Khalil. Nonlinear Systems, MacMillan, USA, 1992.

[5] L. P. Lecoq and A. M. Hopkin. A functional analysis approach to $L_{\infty}$ stability and its applications to systems with hysteresis. IEEETransaction on Automatic Control, vol. AC-17, no. 3, pp. 328-338, 1972.

[6] J. W. Macki, P. Nistri and P. Zecca. Mathematical models for hysteresis. SIAM Review, 35(1), pp. 94-123, 1993.

[7] Y. Q. Ni, J. M. Ko and C. W. Wong. Identification of non-linear hysteretic isolators from periodic vibration tests. Journal of Sound and Vibration, 217(4), pp. 737-756, 1998.

[8] B. F. Spencer. Reliability of Randomly Excited Hysteretic Structures, Springer-Verlag, New York, 1986.

[9] C-Y. Su, Y. Stepanenko, J. Svoboda and T. P. Leung. Robust adaptive control of a class of nonlinear systems with unknown backlash-like hysteresis. IEEE-Transaction on Automatic Control, vol. 45, no. 12, pp. 2427-2432, 2000.

[10] G. Tao and P. V. Kokotovic. Adaptive control of plants with unknown hystereses. IEEE-Transaction on Automatic Control, vol. 40, no. 2, pp. 200-212, 1995.

[11] A. Visintin. Differential Models of Hysteresis, Springer-Verlag, Berlin, Heidelberg, 1994.

[12] Y. K. Wen. Method of random vibration of hysteretic systems. Journal of Engineering Mechanics ASCE, 102(2), 249-263, 1976. 